Revue d'histoire de l'Amérique française

REYUE D.HISTOIRE DE L'AMÉRIQUE FRANÇAISE

\title{
HARVEY, Fernand et Gérard BEAULIEU, dir., Les relations entre le Québec et l'Acadie de la tradition à la modernité (Sainte-Foy/Moncton, Éditions de l'IQRC/Éditions d'Acadie, 2000), 295 p.
}

\section{Nicole Lang}

Volume 56, numéro 1, été 2002

URI : https://id.erudit.org/iderudit/007222ar

DOI : https://doi.org/10.7202/007222ar

Aller au sommaire du numéro

Éditeur(s)

Institut d'histoire de l'Amérique française

ISSN

0035-2357 (imprimé)

1492-1383 (numérique)

Découvrir la revue

Citer ce compte rendu

Lang, N. (2002). Compte rendu de [HARVEY, Fernand et Gérard BEAULIEU, dir., Les relations entre le Québec et l'Acadie de la tradition à la modernité

(Sainte-Foy/Moncton, Éditions de l'IQRC/Éditions d'Acadie, 2000), 295 p.] Revue d'histoire de l'Amérique française, 56(1), 77-81. https://doi.org/10.7202/007222ar d'utilisation que vous pouvez consulter en ligne. 
indépendante puisque l'espace était saturé, mais le travail salarié dans les compagnies minières a constitué une alternative économique incontournable pour une bonne proportion des habitants.

Dans cette étude d'histoire sociale, Donald Dennie combine de façon heureuse l'histoire et la sociologie. Les analyses détaillées des différentes facettes de l'évolution de la population et de ses façons de vivre, de l'économie et des institutions politiques aident à mieux saisir les changements qui se sont produits au fil du temps. De plus, l'auteur illustre ses propos par de nombreux tableaux et témoignages oraux et écrits des différentes époques. L'ouvrage constitue donc un apport important pour la connaissance de l'histoire de la région de Sudbury et il sera très utile à tous ceux qui s'y intéressent.

HÉLÈNE BOIS

Syndicat des chargés de cours

Université Laval

HARVEY, Fernand et Gérard BEAULIEU, dir., Les relations entre le Québec et l'Acadie de la tradition à la modernité (Sainte-Foy/Moncton, Éditions de l'IQRC/Éditions d'Acadie, 2000), 295 p.

Cet ouvrage est l'aboutissement d'un projet de recherche réalisé par une équipe de chercheurs acadiens et québécois sous la direction de Fernand Harvey, professeur titulaire de la Chaire Fernand-Dumont sur la culture, de l'INRS-Culture et Société (Université du Québec) à Sainte-Foy et de Gérard Beaulieu, professeur au département d'histoire et de géographie de l'Université de Moncton. L'objectif central du livre est de présenter une vue d'ensemble de l'évolution des relations Québec-Acadie à partir de la "Renaissance acadienne» jusqu'à nos jours (1880-2000). Puisque ces relations ont connu une série de transformations importantes au cours de la période, les auteurs ont divisé l'ouvrage en deux parties. La première se penche sur l'époque des relations traditionnelles au sein du Canada français, tandis que la seconde présente l'époque de la modernité politique et culturelle contemporaine à partir des années 1960. Ils accordent plus d'importance à l'étude de la période contemporaine, car il y a alors intensification et diversification des relations Québec-Acadie. Selon les auteurs, "la proximité géographique, le partage d'une langue commune et des cheminements historiques tantôt parallèles, tantôt convergents fondent et caractérisent plus que tout autre aspect les relations 
entre le Québec et l'Acadie» (p. 9). Ainsi, le livre accorde une place centrale à la culture par le biais de la langue, de l'historiographie, de la littérature et des arts. Les auteurs souhaitent que leur ouvrage "permette de dépasser les lieux communs sur les relations entre le Québec et l'Acadie et contribue à faire découvrir toute la richesse et la complexité de ces relations en transformation constante» (p. 10).

La première partie du livre, "Les relations traditionnelles entre le Québec et l'Acadie», présente trois chapitres. L'étude de Fernand Harvey se penche sur l'histoire de l'Acadie telle qu'écrite par des historiens canadiens-français avant 1960. Il s'intéresse à l'interprétation que ces historiens faisaient du passé acadien. Harvey démontre que cette historiographie n'est pas aussi homogène qu'on aurait pu le croire. En effet, il existe certains clivages entre les historiens selon qu'ils soient laïques ou religieux, disciples de l'école de Groulx, nationalistes modérés, anglophiles ou encore loyalistes. Le débat entourant la Déportation illustre bien ces clivages. Mais, poursuit Harvey, «il en existe un autre plus subtil qui met en cause les rapports entre les élites québécoises et celles de l'Acadie autour de la question identitaire» (p. 48).

Léon Thériault analyse la question de l'identité acadienne à travers la perception que les élites acadiennes avaient du Québec. Selon l'historien, les éléments clés qui dominent les relations Acadie-Québec entre 1880 et 1960 sont l'affirmation de la nationalité acadienne mais souvent la méfiance des Acadiens à l'endroit du Québec. Ainsi, malgré le fait que l'Acadie a "beaucoup reçu " du Québec, malgré les nombreux contacts et les causes communes, les relations «manquent de chaleur». L'auteur s'interroge sur les origines de cette méfiance. «Faut-il en rechercher l'origine dans la situation de minoritaires des uns et dans celle de majoritaires des autres? Qui nous le dira?» (p. 71).

Neil Boucher s'intéresse aux relations entre les Acadiens de la Nouvelle-Écosse et le Québec, plus particulièrement sur les plans de la religion, de l'éducation et des manifestations patriotiques. Dans le domaine religieux, de nombreux prêtres québécois ont desservi les paroisses acadiennes jusqu'au début $\mathrm{du} \mathrm{xx}^{\mathrm{e}}$ siècle et ont contribué à la formation du clergé acadien. En éducation, le Québec a formé plusieurs générations de professeurs acadiens. De plus, grâce à des bourses d'étude, de jeunes Acadiennes ont pu poursuivre leurs études dans des écoles ou des instituts d'arts ménagers au Québec. Quant aux manifestations patriotiques, l'auteur mentionne particulièrement l'apport financier du Québec au Courrier de la Nouvelle-Écosse. "L’appui matériel et 
moral du Québec, conclut Boucher, est donc venu s'ajouter à une volonté déjà existante sur place, ce qui va mieux préparer les Acadiens de la Nouvelle-Écosse à affronter les défis de survivance linguistique et culturelle que va leur lancer la deuxième moitié du $\mathrm{xx}^{\mathrm{e}}$ siècle» (p. 94).

La deuxième partie, "Le Québec et l'Acadie dans un contexte de modernité», propose huit chapitres et s'ouvre sur une étude de Gérard Beaulieu. Celui-ci étudie les éditoriaux des trois quotidiens acadiens du Nouveau-Brunswick, l'Évangéline, Le Matin et L'Acadie Nouvelle, pour voir l'attitude des éditorialistes à l'égard du Québec. Selon Beaulieu, les attitudes et les prises de position des éditorialistes révèlent que, dans bien des cas, les revendications des Acadiens rejoignent celles des nationalistes québécois.

Yolande Castonguay-Leblanc étudie la contribution du Québec au développement de l'éducation en français au Nouveau-Brunswick. De 1880 à 1960, l'éducation semble être le domaine où les relations QuébecAcadie sont plus importantes. Toutefois, Castonguay-Leblanc s'intéresse plus particulièrement à la situation qui prévaut après 1960. Elle se penche sur plusieurs thèmes dont les rapports entre les associations d'enseignants du secteur public, les programmes d'étude et les méthodes pédagogiques. L'auteure démontre que l'Acadie du NouveauBrunswick a beaucoup profité de tous ces rapports qui, avec les années, se sont transformés en une réelle coopération bilatérale.

Chedly Belkhodja et Roger Ouellette proposent une analyse des relations de coopération entre le Québec et l'Acadie. Depuis 1960, le gouvernement du Québec a négocié et signé des ententes avec les gouvernements des Maritimes sur la langue, les communications et la culture. En 1980, le Québec a ouvert un bureau à Moncton afin de desservir les provinces maritimes puis, à partir de 1984, toute la région atlantique. Belkhodja et Ouellette présentent le contexte de ces événements et évaluent le rôle du bureau du Québec en Acadie.

Maurice Leblanc s'intéresse aux relations culturelles que les Acadiens de la Nouvelle-Écosse entretiennent avec le Québec depuis le début des années 1960. Par la suite, il aborde les disciplines culturelles qui ont bénéficié de l'aide du Québec, soit le théâtre, la musique, le mime, la danse et les arts visuels. L'auteur affirme que "...les Acadiens de la Nouvelle-Écosse ne peuvent que se féliciter de l’appui reçu du Québec, un appui qui a largement contribué au maintien, au développement, à la promotion d'une identité, d'une culture, d'un patrimoine dont la langue française constitue le lien indispensable» (p. 208). 
Le débat sur la langue populaire et le français standard est le sujet de l'étude d'Annette Boudreau et de Mathieu Leblanc. Ils se penchent d'abord sur la querelle du «joual» survenue au Québec entre 1960 et 1975 et ils tentent ensuite d'établir des parallèles avec le débat sur l'emploi du «chiac» dans le Sud-Est du Nouveau-Brunswick de 1970 à 1975. Selon les deux auteurs, l'ampleur des controverses est bien différente d'une province à l'autre mais, dans les deux communautés, la préoccupation semble avoir été la même : élargir le nombre de locuteurs et fournir un véhicule adéquat d'expression de revendications.

Jean Levasseur étudie la réception de la littérature acadienne au Québec depuis 1970. Il démontre jusqu'à quel point l'image de l'Acadie présentée par les médias québécois est ambiguë. Levasseur reproche aux journalistes un «intérêt intermittent», une "paresse journalistique», un "certain impérialisme» qui empêchent les communicateurs québécois de bien saisir la réalité actuelle de l'Acadie. Ces médias véhiculent deux images de l'Acadie: une folklorique et une plus moderne, mais sans faire preuve d'une attitude critique à l'égard de ces deux visions. De plus, ajoute Levasseur, à part quelques exceptions, les collèges et les universités du Québec font trop peu de place à la littérature acadienne.

Fernand Harvey et Christine Duplessis examinent les échanges culturels c'est-à-dire les offres de produits culturels et leur réception dans chacune des communautés. L'étude découle d'entrevues avec près de 20 intervenants: des artistes et des agents culturels du Nouveau-Brunswick, du Québec et de l'Ontario. Ceux-ci soulignent les changements positifs remarqués au cours des dernières années. Ils notent une augmentation et la maturation de la production artistique acadienne, en plus de la mise en place de certaines infrastructures au Nouveau-Brunswick. Par contre, peu d'artistes acadiens peuvent se payer un agent pour s'occuper de l'évolution de leur carrière tant au Québec qu'en Acadie. Au Québec, malgré l'évolution des programmes d'assistance, les artistes acadiens ont beaucoup de difficulté à percer. Les problèmes d'organisation et le manque de connaissance des artistes acadiens de la part du public québécois seraient à l'origine de cette situation.

Le dernier chapitre du livre porte sur le sport, présenté comme un volet des échanges culturels. L'auteur, Jules Lamarre, étudie deux manifestations sportives annuelles importantes: les Jeux du Québec en place depuis 1970 et favorisant la compétition entre athlètes; et les Jeux de l'Acadie qui existent depuis 1980 et qui privilégient une approche parti- 
cipative. Les organisateurs des Jeux de l'Acadie ont bénéficié très tôt de l'expérience du Québec et la collaboration se poursuit d'année en année. Avec le temps, les Jeux de l'Acadie sont devenus une «occasion d'affirmation nationale" pour les jeunes Acadiens et Acadiennes leur permettant ainsi d'acquérir de l'assurance et de la visibilité face à la majorité anglophone. De plus, grâce aux jeux, les Acadiens ont fait des progrès importants sur le plan de l'organisation des divers sports et des installations.

Cet ouvrage collectif a atteint l'objectif de nous faire découvrir toute la richesse et la complexité des relations Québec-Acadie aux $\mathrm{XIX}^{\mathrm{e}}$ et $\mathrm{Xx}^{\mathrm{e}}$ siècles. Il fera désormais référence pour quiconque s'intéresse à cette problématique. Par ailleurs, comme le soulignent les directeurs du projet, "Une meilleure connaissance réciproque est essentielle à la coopération entre Québécois et Acadiens pour le bénéfice des deux groupes et de toute la francophonie nord-américaine.» (p. 16)

NICOLE LANG Campus d'Edmundston Université de Moncton

HOWELL, Colin D., Blood, Sweat, and Cheers. Sport and the Making of Modern Canada (Toronto, University of Toronto Press, coll. «Themes in Canadian Social History », 2001), $161 \mathrm{p}$.

Professeur d'histoire à l'université Saint Mary's, Colin D. Howell est un des rares spécialistes de l'histoire du sport au Canada. Il poursuit ainsi le travail entamé par quelques chercheurs précurseurs, notamment Alan Metcalfe et Bruce Kidd. Son projet est ambitieux. Il s'agit ni plus ni moins que de retracer l'évolution du sport au Canada (l'auteur examine aussi bien le hockey ou le baseball que le rodéo, le cricket et les Jeux olympiques), de la Confédération à aujourd'hui, en cernant les liens entre ce secteur d'activité et les multiples transformations sociales, économiques et politiques ayant affecté le pays. Le cœur de l'ouvrage est centré sur le passage - entre 1867 et la Première Guerre mondiale — de l'amateurisme au professionnalisme, qui a redéfini de façon fondamentale les façons de concevoir l'activité sportive en la transformant en un emploi rémunéré et inséré dans les rapports entre le travail et le capital qui ont accompagné le développement du capitalisme industriel au début du $\mathrm{xx}^{\mathrm{e}}$ siècle. 